\title{
Measuring the Five-Parameter Grain-Boundary Distribution from Observations of Planar Sections
}

\author{
Brent L. Adams \\ b_I_adams@byu.edu \\ Bassem S. El-Dasher \\ Gregory S. Rohrer \\ David M. Saylor
}

Follow this and additional works at: https://scholarsarchive.byu.edu/facpub

Part of the Mechanical Engineering Commons

\section{Original Publication Citation}

Metallurgical and Materials Transactions Jul 24 35A, 7 Research Library pg. 1981

\section{BYU ScholarsArchive Citation}

Adams, Brent L.; El-Dasher, Bassem S.; Rohrer, Gregory S.; and Saylor, David M., "Measuring the FiveParameter Grain-Boundary Distribution from Observations of Planar Sections" (2004). Faculty Publications. 425.

https://scholarsarchive.byu.edu/facpub/425 


\title{
Measuring the Five-Parameter Grain-Boundary Distribution from Observations of Planar Sections
}

\author{
DAVID M. SAYLOR, BASSEM S. EL-DASHER, BRENT L. ADAMS, \\ and GREGORY S. ROHRER
}

\begin{abstract}
A stereological method is described for estimating the distribution of grain-boundary types in polycrystalline materials on the basis of observations from a single planar section. The grain-boundary distribution is expressed in terms of five macroscopically observable parameters that include: three parameters that describe the lattice misorientation across the boundary and two parameters that describe the orientation of the grain-boundary plane normal. The grain-boundary distribution is derived from measurements of grain orientations and the orientations of the lines formed where grain boundaries intersect the plane of observation. Tests of the method on simulated observations illustrate that the distribution of boundaries in a material with cubic symmetry can be reliably detcrmined with about $10^{\circ}$ of resolution from the analysis of $5 \times 10^{4}$ or more line segments. Furthermore, grain-boundary distributions directly observed from serial sections of a $\mathrm{SrTiO}_{3}$ polycrystal are compared to those resulting from the stereological analysis of a single plane. The comparison shows that the stereological method provides a reasonable estimate of the measured distribution. The differences between the directly observed grain-boundary distribution and that derived from the stereological analysis are consistent with the results from the simulation.
\end{abstract}

\section{INTRODUCTION}

INTERFACES between grains or phases in threedimensional microstructures are typically observed as line segments on planar sections. While X-ray diffraction microscopy ${ }^{11 !}$ and serial sectioning ${ }^{21}$ are capable of providing complete three-dimensional characterization, each technique poses experimental challenges that have, at least until now, prevented their widespread application. In a previous article, a technique that used observations from a planar section to determine the average three-dimensional habits of fully faceted crystals embedded in a second phase was described. ${ }^{[3]}$ This method, adapted from established stereological methods, ${ }^{14,5]}$ enables a statistical description of crystal habits to be determined from geometric information in conventional micrographs, along with the crystal orientation data obtained from electron back-scattered diffraction patterns (EBSPs) in a scanning electron microscope. ${ }^{|6|}$ The purpose of this article is to demonstrate that the method developed for fully faceted crystals can be expanded to the more complex problem of extracting a continuous distribution of grain-boundary planes, specified by area fraction, from a single planar section through a single-phase polycrystalline material.

The grain-boundary distribution, $\lambda(\Delta g, \mathbf{n})$, is defined as the relative frequency of occurrence of a grain boundary

DAVID M. SAYLOR, Postdoctoral Research Associate, is with the National histitute of Standards and Technology, Gaithersburg, MD 20899. Contact e-mail: david.saylor@nist.gov BASSEM S. EL-DASHER, Postdoctoral Research Associate, is with the Lawrence Livermore National L aboratory, Livermore, CA 94550. BRENT L. ADAMS, Professor, is with the Department of Mechanical Engineering, Brigham Young University, Provo, UT 84602. GREGORY S. ROHRER, W.W. Mullius Professor, is with Department of Materials Science and Engineering, Carnegic Mellon University, Pittsburgh, PA 15213.

This article is based on a presentation made at the symposium "Characterization and Representation of Material Microstructures in 3-D" held October 8 - 10,2002 , in Columbus, $\mathrm{OH}$, under the auspices of ASM International's Phase Transformations committee. with a misorientation, $\Delta g$, and boundary plane normal, $\mathbf{n}$, in units of multiples of a random distribution (MRD). Each grain boundary therefore has five parameters, three that describe the misorientation and two that describe the boundary plane normal. Observations in single section planes can be used to specify four of the five parameters. The only unknown parameter is the inclination angle between the observation plane and the grain boundary plane. While this angle cannot be determined from a single section plane, we do know that the true boundary plane must be in the set of planes whose normals are perpendicular to the observed boundary line segment and trace out a great circle on a stereographic projection.

This information is used in our analysis in the following way. We make observations of many crystal pairs that have indistinguishable misorientations, but are oriented differently with respect to the section plane. The observed line segment associated with each of these bicrystals generates a set of possible boundary planes. The probability that each set contains the true boundary plane is one, and the probability that any arbitrary plane is included in the set is less than one. Therefore, after many observations of the same type of bicrystal, true boundary planes make up a relatively larger fraction of the accumulated data than the arbitrary planes, and can therefore be identified as peaks in the distribution. To quantify the areal distribution of the boundary planes, the inhomogeneous background caused by the incorrect planes in each set must be removed. Here, we use an approximate method, based on the assumption of random sampling, to remove the incorrectly assigned lengths. After this correction, the ratios of the observed line lengths allows us to specify the relative areas of each boundary type and, therefore, the five parameter distribution, $\lambda(\Delta g, \mathbf{n})$.

In Section II, the details of the method for estimating $\lambda(\Delta g, \mathbf{n})$ from observations in a planar section are described. The method is validated by tests on simulated data sets where the true grain-boundary distribution is known, as well as an 
experimental data set where the grain-boundary distribution has been measured by direct observation. The generation of these data sets is described in Section III. In Section IV, the results of the stereological analysis are quantitatively compared to the simulated data set and the experimental data set. In Section V, we discuss possible applications of the method, some inherent crrors, and suggest potential improvements. The article concludes with a summary.

\section{METHOD}

Consider a two-dimensional section through a polycrystalline material, where the grain-boundary traces, $\mathbf{I}_{i j}^{\prime}$, and grain orientations, $g_{i}$, are known (Figure 1). Note that throughout the manuscript, vectors and angles in the sample reference frame are indicated by a prime and those in a crystallographic reference frame are unprimed. Here, $g$ is defined as a transformation (passive rotation) of an orthogonal coordinate system, written as an orthogonal matrix (with determinant = +1 ), that describes the transformation between the fixed sample coordinate system $\left(\mathbf{e}_{i}^{\prime}\right)$ and the crystal coordinate system. Every observed grain-boundary trace $\mathbf{l}^{\prime}$ is associated with two grain orientations $\left(g_{i}\right.$ and $\left.g_{j}\right)$, which can be used to calculate the lattice misorientation across the boundary $\left(\Delta g_{i j}\right)$ and a radial angle $\left(\phi^{\prime}\right)$ in the plane of observation (Figure 1 ). Although we do not know how the grain boundary is inclined with respect to the observation plane, we do know that it belongs to a sel of planes that includes the surface trace and obeys the condition $\mathbf{l}_{i j}^{\prime} \cdot \mathbf{n}_{i j k}^{\prime}=0$, where the vectors $\mathbf{n}_{i j k}^{\prime}$ are unit normals to the planes. In other words, the true grainboundary plane must be in the zone of $\mathbf{1}_{i j}^{\prime}$. For each misorientation, sets of $\mathbf{n}_{i j k}^{\prime}$ are accumulated and weighted according to the length of the observed boundary trace, $\left|\mathbf{I}_{i j}^{\prime}\right|$.

A discrete set of grain-boundary types covering the range of macroscopic parameters is used as an approximation of the continuous distribution $\lambda(\Delta g, \mathbf{n})$. Every possible observed

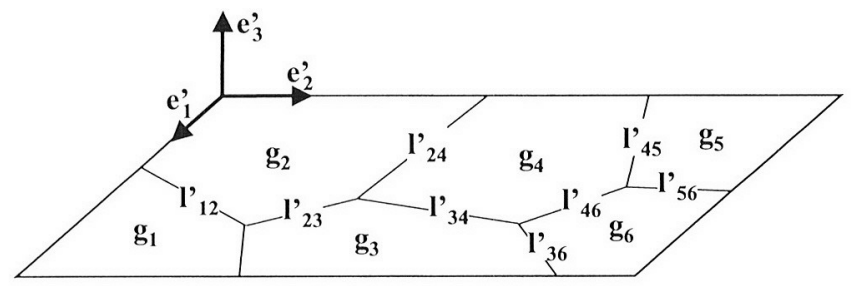

(a)

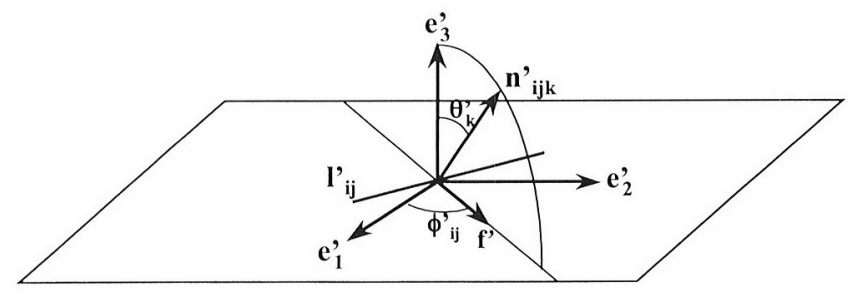

(b)

Fig. 1-(a) Observation of the sample in the laboratory reference frame. The polygons are planar sections of grains in a polycrystalline material with known orientations, $g_{i}$. (b) Definition of the variables in the laboratory reference frame. The normal vectors to the possible bounding planes, $\mathbf{n}_{i j k}^{\prime}$, trace out a circular are. plane, $\mathbf{n}_{i j k}$, is added to a cell in this discrete data structure, and therefore, we refer to it as an accumulator array. The misorientation is specified by three Eulerian angles $\left(\phi_{1}, \Phi, \phi_{2}\right)$ and the boundary plane by two spherical angles, $\theta$ and $\phi$, in a crystallographic coordinate system. The domain of misorientations was parameterized by $\phi_{1}, \cos \Phi$, and $\phi_{2}$, and the spherical angles were parameterized by $\cos \theta$ and $\phi$. This particular parameterization was used because it yields cells of equal volume when the parameters are equally partitioned. In this article, we consider only materials with cubic symmetry. Because crystal symmetries give rise to numerous indistinguishable misorientations, this allows us to limit the range of the boundary parameters. ${ }^{71}$ For computational ease, we have defined the accumulator over a range of zero to $\pi / 2,1, \pi / 2$, and $\pi$ for the boundary parameters $\phi_{1}, \cos \Phi, \phi_{2}$, and $\phi$, respectively; $\cos \theta$ is in the range of -1 to 1 . For a material with cubic symmetry, this volume of the boundary space contains 36 symmetrically equivalent asymmetric domains, which are domains that contain a complete and unique set of physically distinct grain boundaries. For the work described here, we have partitioned the range of misorientation parameters $\left(\phi_{1}, \cos (), \phi_{2}\right)$ into nine equal cells and the range of boundary plane parameters $(\phi, \cos \theta)$ into 18 equal cells. Thus, the resolution of the discretization is approximately $10 \mathrm{deg}$.

The accumulator is filled according to the following procedure. First, for each observed line segment, the adjacent orientations, $g_{1}$ and $g_{2}$, are used to calculate the misorientation, $\Delta \mathrm{g}=\mathrm{g}_{1} \mathrm{~g}_{2}{ }^{T}$. Next, each plane in the set $\mathbf{n}_{i j k}^{\prime}$ is parameterized by two spherical angles, $\phi_{i j}^{\prime}$ and $\theta_{k}^{\prime}$. The in-plane angle, $\phi_{i \mathrm{ij}}^{\prime}$, is characteristic of all planes in a set arising from a single observed line segment $\mathbf{I}_{i j}$. The values of the azimuthal angle, $\theta_{\mathrm{k}}^{\prime}$, are in the range $-\pi / 2 \leq \theta \leq \pi / 2$ and take $B$ discrete values. The range for each vector type is $(1 / B) \cos \theta$, and we have most frequently used $B=90$, although the results were insensitive to the choice of $B$, for $B \geq 15$. The normal vector in the sample reference frame, $\mathbf{n}_{i j k}^{\prime}$, parameterized by $\phi_{\mathrm{ij}}^{\prime}$ and $\theta_{\mathrm{k}}^{\prime}$, is then rotated to the crystal reference using the nontransposed orientation, $\mathbf{n}=g_{1} \mathbf{n}^{\prime}$. The values of $\Delta g$ and $\mathbf{n}$ identify the cell in the accumulator to which the length of an observed boundary trace is added. Note that crystal symmetries lead to numerous values of $\Delta g$ that represent indistinguishable bicrystals. Each of these equivalent representations is considered and added to the accumulator. This includes the equivalency of expressing the grainboundary plane normal in terms of one crystal or the other. In other words, for each observation, equivalent representations expressed in terms of both crystals are added to the accumulator. The procedures used to determine the symmetrical equivalents are described in detail elsewhere. ${ }^{181}$ This entire procedure was repeated for the $B$ possibilities of $\theta^{\prime}$ for each line segment, and then for all line segments in the datal set.

Recall that the probability that each set of plane normals, $\mathbf{n}_{i j k}^{\prime}$, contains the true boundary plane is one, and the probability that an arbitrary incorrect plane is included is less than one. Therefore, after many observations, the cells in the accumulator corresponding to true boundary planes will have values that are larger than those corresponding to incorrectly assigned planes. Ideally, we would like to know the fractional length of line segments crossing each plane, since this is equal to the fractional area of each plane. ${ }^{\mid 91}$ However, it is clear that too much length has been added to the accumulator. 
Furthermore, it is obvious that the error is not uniformly distributed. For example, consider observations of line segments arising from grain boundaries with the same misorientation. The domain of possible discrete boundary plane orientations in the crystal reference frame at a fixed misorientation forms a $D \times D$ matrix, where $D$ is the number of divisions of the accumulator in the range of $-1 \leq \cos \theta \leq 1$ and $0 \leq \phi \leq \pi$; here, $D$ was chosen to be 18 . If there is a peak in the real distribution of grain-boundary planes, then orientations very close to this pole will have more incorrectly assigned length than others. This is because these neighboring cells have a greater probability of being in the zone of the peak orientation than an orientation further away. Conversely, orientations far from the maximum are less likely to share a zone with the most highly populated orientation and will accumulate less incorrectly assigned length. This can be quantified in the following way. In the $i$ th boundary plane cell for a particular misorientation, there is an observed length, $l_{i}{ }^{\circ}$. This is the sum of the length correctly assigned to this cell $\left(l_{i}^{c}\right)$ and the length that was correct in some other cell, $j$, but was assigned to $i$ because $i$ and $j$ were both in the zone of the observed trace. Therefore, the total length is

$$
l_{i}^{o}=l_{i}^{c}+\sum_{j=1, j=i}^{D^{2}} \omega_{i j} l_{j}^{c}
$$

where $\omega_{i j}$ is a weighting factor that describes the fraction of great circles whose true plane is $j$, but also pass through $i$ because they are in the same zone. The factor $\omega_{i j}$ is largest when $i$ and $j$ are neighboring cells and decreases to a constant as the angular separation between $i$ and $j$ increases. This suggests a method for determining an approximate solution. First, we will estimate the average value of the incorrectly assigned length that originated from distant (nonnearest neighbor) cells and subtract this from the $i$ th cell. Second, we approximate the incorrectly assigned length that originated in nearest neighbor cells and subtract this quantity. These two quantities will be an approximation for the sum on the right-hand side (RHS) of Eq. [1]. In the following paragraphs, we describe the approximate solution and the assumptions required. The validity of the assumptions are left for the discussion in Section V.

The average value of the incorrectly assigned length from distant cells can be calculated by making the approximation that the correctly assigned length in the $j$ th cell $\left(l_{j}^{c}\right)$ is equal to the average correct length in each box $\left(\left\langle l^{c}\right\rangle=l^{c} / D^{2}\right)$. Note that this approximation is only correct if the boundary planes are randomly distributed. With this approximation, we now have

$$
\sum_{j=1}^{D^{2}} \omega_{i j} l_{j}^{c} \approx \sum_{j=1}^{D^{2}} \omega_{i j}\left\langle l^{c}\right\rangle
$$

where the term on the RHS is the average incorrect length assigned to each cell. Note that for any length of line added to the accumulator, the ideal probability of a correct assignment is $1 / D$ and the ideal probability of an incorrect assignment is $(D-1) / D$. In fact, because the domain of possible plane normals is discretized using an equal area scheme, there is not a one-to-one mapping between cells in the laboratory frame and the crystal frame, and this alters these probabilities. Nevertheless, we will assume these probabilities to be acceptable. Therefore, the average of the incorrect length assigned to a given cell can also be written in the following way:

$$
\sum_{j=1}^{D^{2}} \omega_{i j}\left\langle l^{c}\right\rangle=\frac{(D-1)}{D}\left\langle l^{\circ}\right\rangle
$$

Finally, since the ratio of $\left\langle l^{\circ}\right\rangle$ to $\langle l\rangle$ is $D$,

$$
\sum_{j=1}^{D^{2}} \omega_{i j}=(D-1)
$$

The quantity in Eq. [3] is the correction from all of the cells. However, some fraction of this, which we will define as $Z$, comes from the nearest neighbor cells. Because we intend to account for this contribution separately, it should be subtracted from the total. With this final consideration, the contribution from distant cells is

$$
\sum_{j=N N+1}^{D^{2}} \omega_{i j}\left\langle l^{c}\right\rangle=(1-Z) \frac{(D-1)}{D}\left\langle l^{\circ}\right\rangle
$$

where $N N$ is the number of near neighbor cells. We can now rewrite an approximation for Eq. [1] as

$$
l_{i}^{c} \approx l_{i}^{\circ}+(Z-1) \frac{(D-1)}{D}\left\langle l^{\circ}\right\rangle-\sum_{j=1}^{N N} \omega_{i j} l_{j}^{c}
$$

Note that if the distribution is smooth and continuous, and $D$ is large enough, then the correct length in the $i$ th cell should be approximately equal to the correct length in the adjacent (jth) cell. Using this substitution and taking the sum over the neighboring values of $\omega_{i j}$ to be $Z(D-1)$, from Eq. [4], Eq. [6] can be rewritten in the following way:

$$
l_{i}^{c} \approx l_{i}^{0}+(Z-1) \frac{(D-1)}{D}\left\langle l^{\circ}\right\rangle-Z(D-1) l_{i}^{c}
$$

Rearranging, we reach the following expression:

$$
l_{i}^{c}=\frac{l_{i}^{\mathrm{o}}+(Z-1) \frac{(D-1)}{D}\left\langle l^{\circ}\right\rangle}{1+Z(D-1)}
$$

Taking $Z$ to be the fraction of concentric circles around the point of interest that falls within an angular range of $\pi / 2$, then $Z=2 / D$. Therefore, using Eq. [8], we can estimate the correct length for each boundary plane cell at any given misorientation. Once the correction has been applied at each misorientation, the area, estimated by the total lengths, in each cell is normalized such that the average value is one. Thus, because the cells are of equal volume, the cell values now represent MRD, where the MRD of a given cell is the ratio of area observed to the area expected if the distribution of boundaries was completely random. For any specified grain-boundary type, the MRD value is determined by averaging the MRD values of the corresponding 36 symmetrically equivalent cells within the accumulator. 


\section{TRIAI, DATA SETS}

\section{A. Simulated Data}

The best way to validate the method is to use grain-boundary line segments where the actual grain-boundary planes are known. Thus, we have created simulated data sets consisting of grain boundaries generated according to a target distribution. Becaluse we have gencrated all five parameters, the result we should obtain, $\lambda\left(\Delta_{g}, \mathbf{n}\right)$, is known from the start. Each of these boundaries is then randomly orientated with respect to a virtual observation plane. The trial data is then limited to only the line segments along which the boundaries intersect the observation plane and the orientations of the adjacent grains. To these data, we then apply the stereological procedure to recover an estimate of $\lambda(\Delta g, \mathbf{n})$.

To generate the trial grain-boundary geometries, we use a hypothetical distribution that is representative of those observed in actual polycrystals. Previously, $\mathrm{MgO}$ was the only material in which the complete five parameter grainboundary distribution had been measured. ${ }^{|8|}$ The overwhelming trend in the $\mathrm{MgO}$ distribution was a strong preference for boundaries with a $<100>$ type boundary plane. Thus, we have incorporated a preference for a particular boundary plane type at all fixed misorientations into the simulated data. We use a hypothetical function with maxima in $\lambda(\Delta g, \mathbf{n})$ for boundaries with $\langle 111\rangle$ type boundary planes at all fixed misorientations. To specify the function, we start with the same discrete model for $\lambda(\Delta g, \mathbf{n})$ as used for the accumulator described in Section II. To assign a frequency value, $F$, to each cell in the five-parameter space, we calculate $\theta_{111}$, defined as the minimum angle between the boundary plane normal (taken at the center of the cell) and a $\langle 111\rangle$ direction. We then assign the frequency in the following way:

$$
F=A \exp \left(-\left(\frac{\theta_{111}}{B}\right)^{2}\right)+C
$$

where $A, B$, and $C$ were set to 10,15 , and 1 , respectively. Thus, grain boundaries with plane normals near $\langle 111\rangle$ will have larger assigned frequencies than those away from $\langle 111\rangle$. After a value is assigned to each cell, it is normalized so that the sum of the values in all of the cells is equal to one. Thus, the value assigned to each cell represents the probability with which grain boundaries in the cell occur.

Random grain boundaries were generated by selecting random misorientations, represented by a set of three Euler angles $\left(\phi_{1}, \Phi, \phi_{2}\right)$. To ensure that all possible misorientations are selected with equal probability, values of $\phi_{1}$ and $\phi_{2}$ were selected randomly in the range of 0 to $\pi / 2$ and a value of $\cos \Phi$ was selected in the range 0 to 1 . Random boundary plane normals were generated in an analogous manner. The boundary is added to the appropriate cell based on a conditional probability: if a randomly generated number between zero and one is less than the frequency assigned to the cell, the boundary is accepted. Otherwise, the boundary is discarded. This process is repeated until the desired number of grain boundaries have been accepted and included in the simulated data set.

To randomly orient each boundary with respect to a virtual sample reference frame, a random orientation, $g_{1}$, is generated for one of the grains adjacent to the boundary. Note that with $g_{1}, \Delta g$, and $\mathbf{n}$ lixed, $g_{2}$ and $\mathbf{n}^{\prime}$, the direction of the boundary plane normal in the sample reference frame, are also fixed
The boundary trace, $l^{\prime}$, is assumed to have unit length and its components on the virtual observation plane are $l_{1}^{\prime}=-n_{2}^{\prime} / \sqrt{\left(n_{1}^{\prime 2}+n_{2}^{\prime 2}\right)}, l_{2}^{\prime}=n_{1}^{\prime} / \sqrt{\left(n_{1}^{\prime 2}+n_{2}^{\prime 2}\right)}$, and $l_{3}^{\prime}=0$. Finally, only the orientations of the adjacent grains and the boundary trace direction are recorded and this corresponds to a single simulated observation. After this process is repeated for each boundary, we are left with a simulated data set that mimics one that can be generated experimentally. To test the influence of the number of line segments on the performance of the stereological procedure, simulated data sets consisting of $1 \times 10^{4}, 5 \times 10^{4}, 1 \times 10^{5}$, and $2.5 \times 10^{5}$ grain boundaries were generated. In each case, the stereologically derived grainboundary distribution was compared to the known values of $\lambda(\Delta g, \mathbf{n})$.

\section{B. Experimental Data}

In a previous article, ${ }^{|8|}$ we described a technique that combines conventional microscopy and automated EBSP collection with serial sectioning to characterize the three-dimensional geometry and crystallography of a large number of contiguous crystallites in a polycrystalline material. Information from parallel section planes through the microstructure is used to specify the structure of the interlace network, and ultimately, $\lambda(\Delta g, \mathbf{n})$. Here, we have used this procedure, with minor changes, to characterize a volume of material within a $\mathrm{Sr}^{T} \mathrm{TiO}_{3}$ polycrystal. The grain-boundary distribution will be analyzed and described in detail in a forthcoming article. Here, the observations are compared to the results of a stereological analysis of the same data. A synopsis of the experimental procedure is provided in the remainder of this section.

The $\mathrm{SrTiO}_{3}$ polycrystal used in this study was made by uniaxial compression of 99 pet pure $\mathrm{SrTiO}_{3}$ powder (Aldrich Chemical Company, Inc., Milwaukee, WI)! ${ }^{10 \mid}$ The sample was heated in vacuum $\left(25 \mathrm{~mm} \mathrm{Hg}\right.$ ) at $800^{\circ} \mathrm{C}$ for 25 hours. In a reconditioned airflow, the temperature was then increased at $3{ }^{\circ} \mathrm{C} / \mathrm{min}$ until is reached $1350{ }^{\circ} \mathrm{C}$, and it was then annealed for 10 hours. To enlarge the grains, the sample was heated in air at $1650^{\circ} \mathrm{C}$ for 20 hours. To reveal the positions of the grain boundaries, the polished sample was thermally etched at $1400^{\circ} \mathrm{C}$ for 6 minutes in air. The grain-boundary configurations were determined with a combination of optical images and EBSPs acquired at regular intervals on the sample surface. We started by collecting slightly overlapping grids of optical images on a planar section of the material. Here, five grids consisting of $6 \times 6$ images with a pixel-to-pixel resolution of $0.9 \mu \mathrm{m}$ were collected using an optical microscope. The total area was approximately $0.4 \mathrm{~cm}^{2}$. After a thin carbon coating was applied to eliminate charging, the sample was placed in a PHILIPS* XL40 field emission gun (FEG)

PHIL IPS is a tradenark of Philips Electronic Instruments, Mahwah, NJ.

scanning electron microscope (SEM) and EBSPs were collected at intervals of $20 \mu \mathrm{m}$ over the same areas on the sample surface covered by the optical images. The EBSPs were indexed with TexSEM Laboratories, Inc. (Draper, UT), Orientation Imaging Microscopy (OIM) software version 3.03 , which resulted in a set of Euler angles $\left(\phi_{1}, \Phi, \phi_{2}\right)$ relating the sample reference frame to the crystal reference frame at each probe point. After scanning was completed, $5.2 \pm$ $0.3 \mu \mathrm{m}$ of material was removed using an automatic polisher 
(Logitech PM5, Glasgow, Scotland). The same areas on the sample surface were then located on the new layer and the entire process was repeated. The EBSP data indicated that the sample had no significant grain orientation texture.

The information from the optical images and the EBSP maps were combined to form high-resolution orientation maps from two parallel section planes. First, to accurately specify the crystallite geometry, we manually digitized the grain boundaries in each optical image using a program that allows the operator to trace the boundaries with a computer mouse. This process resulted in binary images of the grain-boundary skeleton. Next, we combined the 36 binary images from each $6 \times 6$ scan area using the positions provided by the optical microscope stage coordinates, creating large image montages of the grainboundary skeleton in each area. The final step was to combine the image montages with the orientation data. For each grain, identified by contiguous pixels not associated with a grain boundary, an orientation was assigned manually by visually matching the grain with its corresponding EBSPs. More than 5000 distinct grains were identified in this way. At this stage, the information required for input into the stereological analysis could be extracted from the orientation maps. Every fourth pixel along a boundary served as an end point for a boundary line segment. Note that the resolution limit of the segmentation scheme is about $4 \mu \mathrm{m}$ ( 4 pixels $\bullet 0.9 \mu \mathrm{m} / \mathrm{pixel}$ ). Any nonlinearity in the boundary geometry below the resolution limit is ignored. Thus, any tendency for a boundary to form facets below this limit will be neglected. Although there were approximately $1 \times 10^{5}$ of these segments, many of these are indistinguishable observations, formed when a linear segment is partitioned into smaller segments. We estimate that the $1 \times 10^{5}$ segments characterized actually consisted of only about $3 \times 10^{4}$ distinguishable segments broken down into smaller elements.

For a comparison, we can use the three-dimensional information from the experiment to extract the true distribution of boundaries. The initial step is to align the parallel sections so that they are fixed in a single reference frame. This was accomplished using a transformation that maximizes the area of overlap between positions in adjacent layers having the same orientation. Next, parts of single grains on both planar sections were identified by comparing the spatial overlap of grains with similar orientations. Common grain boundaries on the two layers were then identified and used to create a meshed interfacial surface. Again, we considered every fourth pixel on the boundaries, except now they served as the vertex of one of the $1 \times 10^{5}$ triangular elements of the mesh. Here, each element represents an observed grain-boundary plane segment for which the misorientation and boundary plane are known. These segments allowed us to specify the true distribution of boundaries in the sample volume using the previously described discrete model for $\lambda(\Delta g, \mathbf{n})$.

\section{RESULTS}

We first consider the analysis of the simulated data sets. To examine the five dimensional $\lambda(\Delta g, \mathbf{n})$, we choose a fixed $\Delta g$ in the three-dimensional misoricntation space, described here in terms of an axis-angle pair, and then plot $\lambda(\mathbf{n})$ on a stereographic projection. For example, Figure 2 compares the stereologically determined distribution of boundary plane normals

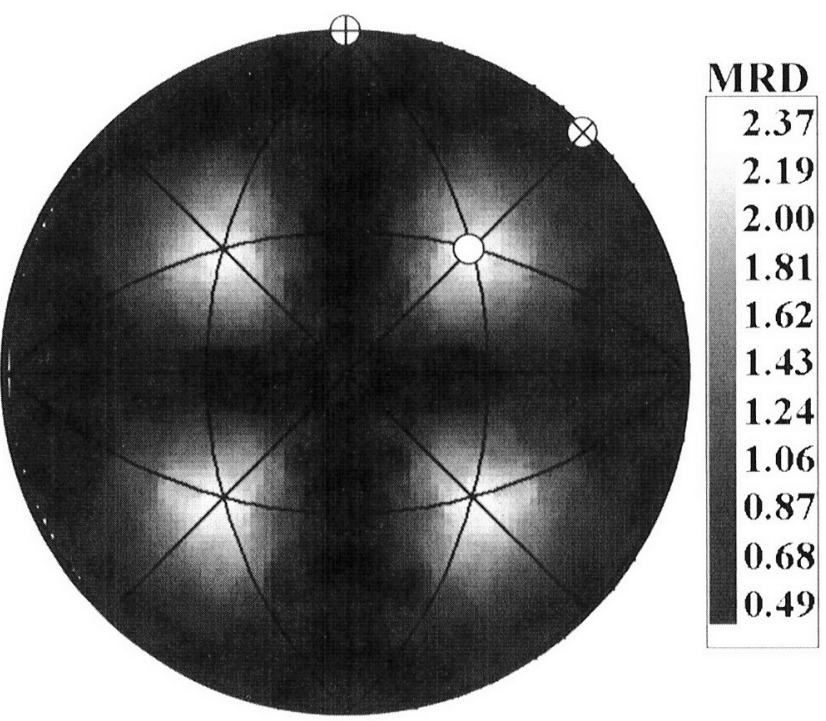

(a)

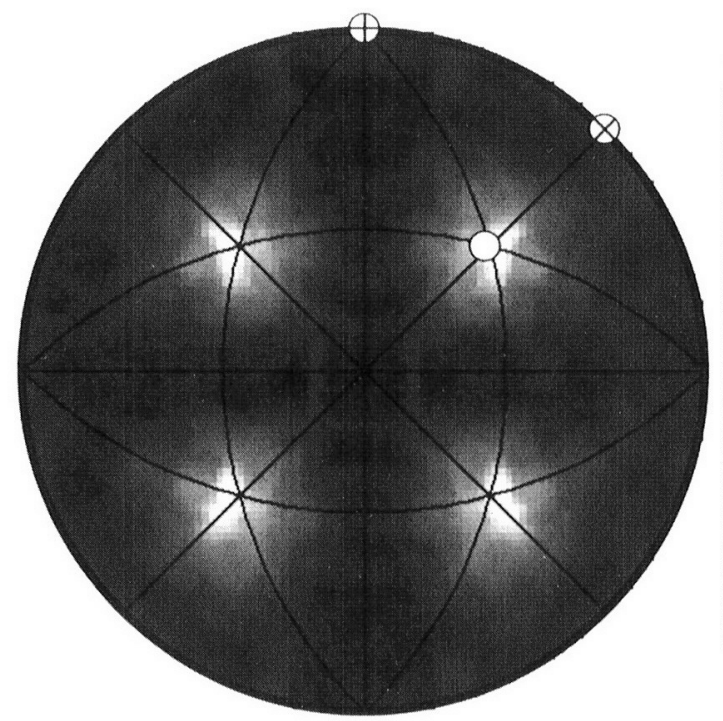

MRD

2.81

2.58

2.35

2.12

1.90

1.67

1.44

1.22

0.99

0.76

0.53

Fig. 2 - (a) and (b) The estimated distribution of grain-boundary plane normals based on $5 \times 10^{4}$ simulated boundaries with a misorientation of $5 \mathrm{deg}$ about $[100]$. For comparison, the actual distributions of plane normals is shown in (b). The populations, represented as MRD, are plotted in stereographic projection, with the [010], [110], and [111] directions marked with a circled "+," and a circled " $\times$," and an open circle, respectively.

(a) to the actual distribution (b) for a fixed misorientation of 5 deg about $[100 \mid$. In this plot, the misorientalion axis is in the plane of the paper, in the $|100|$ direction. Both plots have peaks for plane orientations at $\langle 111\rangle$ and minima along the zone of pure tilt boundaries (these are the boundaries with normals perpendicular to the misorientation axis that lie along the great circle that goes through $[0|0|, \mid 00) 1 \mid$, and $|0| \overline{|0|} \mid$ ). Figure 3 shows the distribution recovered from $5 \times 10^{4}$ observations at three additional points in the high-angle region of misorientation space. The projections in Figures 3(a) through (c) show the plane distributions for 15,30 , and 4.5 (leg about $[10(0) \mid$, with the actual distributions in Figure 3(d) through (I). In cach case, the plane distributions estimated with our algorithm are visually similar to the actual underlying distributions. 

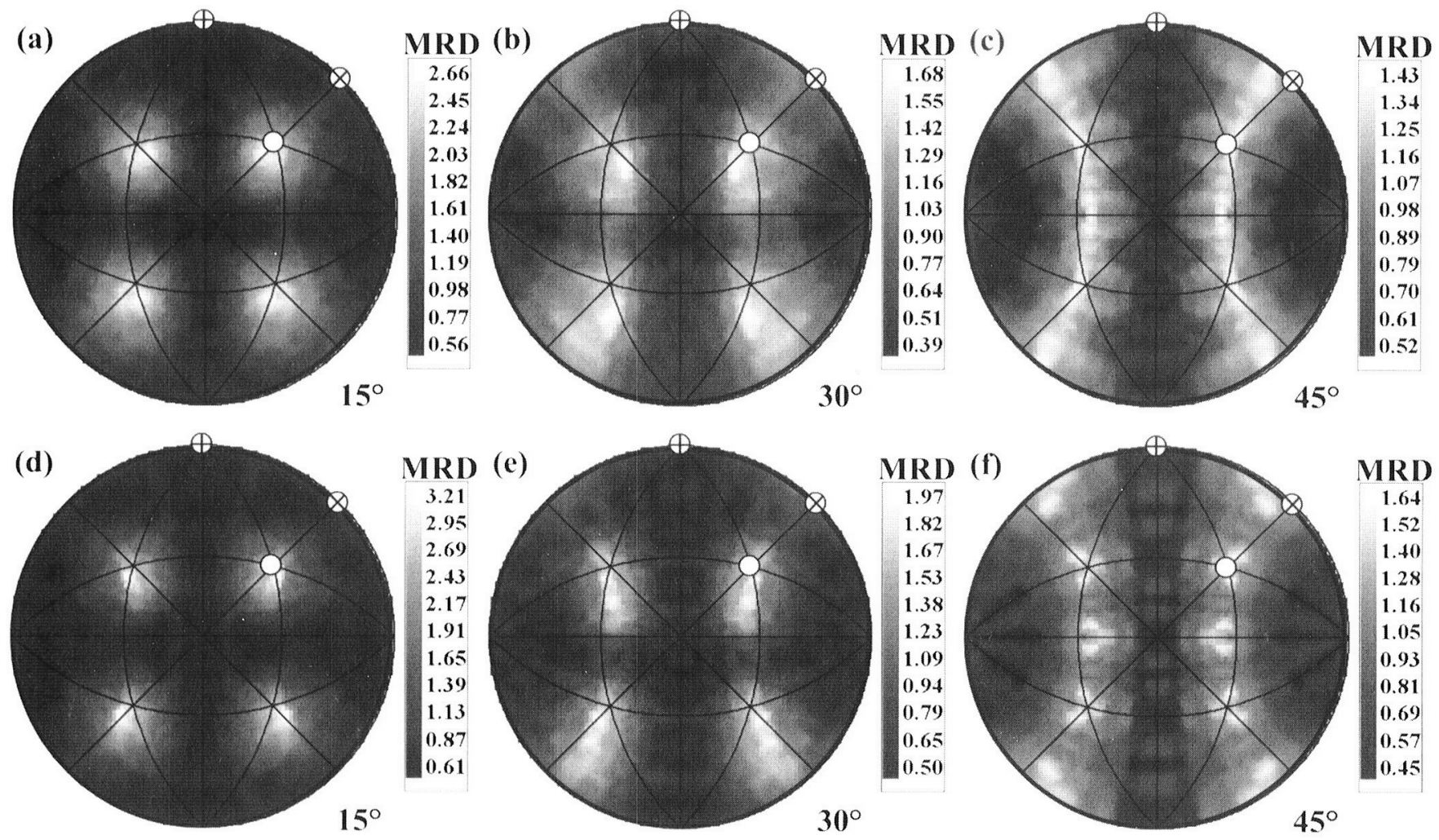

Fig. 3-The distribution of grain-boundary plane normals, recovered using our method on a data set consisting of $5 \times 10^{4}$ simulated boundaries, for boundaries with misorientations of $(a) 15 \mathrm{deg},(b) 30 \mathrm{deg}$, and $(c) 45 \mathrm{deg}$ about [100]. For comparison, the actual distributions of plane normals in the simulated data set for the respective misorientations are shown in $(d)$ through $(f)$. The populations, represented as MRD, are plotted in stereographic projection, with the $[010],[110]$, and $[111]$ directions marked with a circled "+," a circled " $\times$," and an open circle, respectively.

Although the similarity of the actual and estimated distributions is obvious, the plots in Figures 2 and 3 represent only a small fraction of the entire live-dimensional grain-boundary space. Therefore, we have defined a quantity to represent the error, $\delta$, which is the absolute value of the difference between the estimated and actual distribution at a given cell of the live-parameter space. By examining the distributions of this error, we can assess the overall performance of the method. The distribution of error for the case of $5 \times 10^{+}$simulated grain boundaries is shown in Figure 4 as the finely dashed line. We lind that the errors have a maximum at zero MRD of about $27 \mathrm{pet}$ of all boundary types. In other words, zero MRD is the most frequent difference between the observed and actual boundary distributions. The error then decreases steadily along the abscissal, approaching zero at about 1.00 MRD. Further, we have determined that 95 pet of the errors fall between zero and about 0.60 MRD. Considering the fact that the trends in the stereologically derived distribution mimic those in the real distribution, we consider this level of error to be acceplable.

Tests on the other simulated data sets yielded predictable results. The distribution of errors for the data sets consisting of $1 \times 10^{+}, 1 \times 10^{5}$, and $2.5 \times 10^{5}$ are also shown in Figure 4. From the plot, we see that as the number of boundaries in the data set increases, the peak in the distribution at zero MRD markedly increases and the rate of decrease along the abscissa increases, i.e., the width of the distribution contracts. We have determined that 95 pet of the errors are contained within the range of zero and approximately $1.1,0.48$,

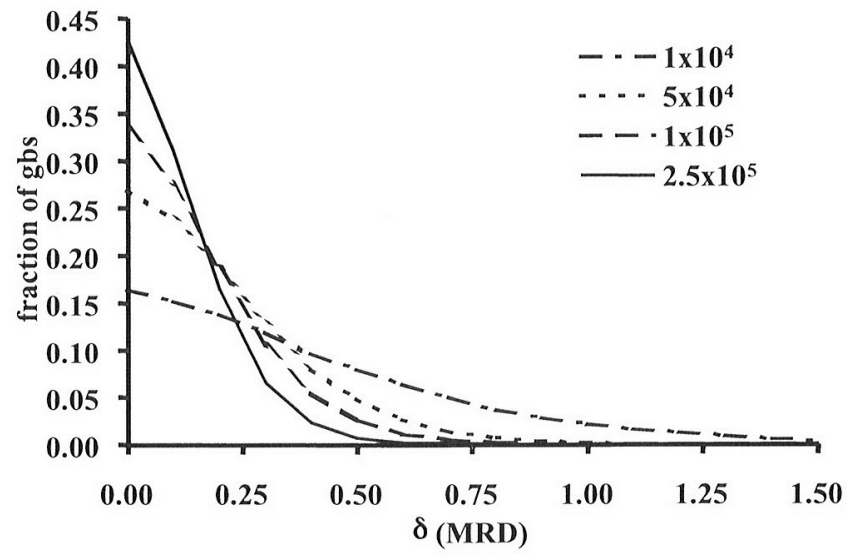

Fig. 4-The distribution of the differences (absolute value) between the recovered and actual grain-boundary distributions from data sets consisting of $1 \times 10^{4}, 5 \times 10^{4}, 1 \times 10^{5}$, and $2.5 \times 10^{5}$ simulated boundaries.

and $0.36 \mathrm{MRD}$ for data sets consisting of $1 \times 10^{4}, 1 \times 10^{5}$ and $2.5 \times 10^{5}$ simulated grain boundaries, respectively. Based on the figure, we observe that with only $1 \times 10^{4}$ observations, there are significant errors $(>0.50 \mathrm{MRD})$ in a large fraction of the cells. However, as more observations are added, the fraction of cells with large crrors quickly goes to zero. In fact, the errors associated with the method do not change substantially after about $5 \times 10^{4}$ observations.

The analysis was also applied to the experimental measurements of $\mathrm{SrTiO}_{3}$. The plot in Figure 5(a) shows the boundary 

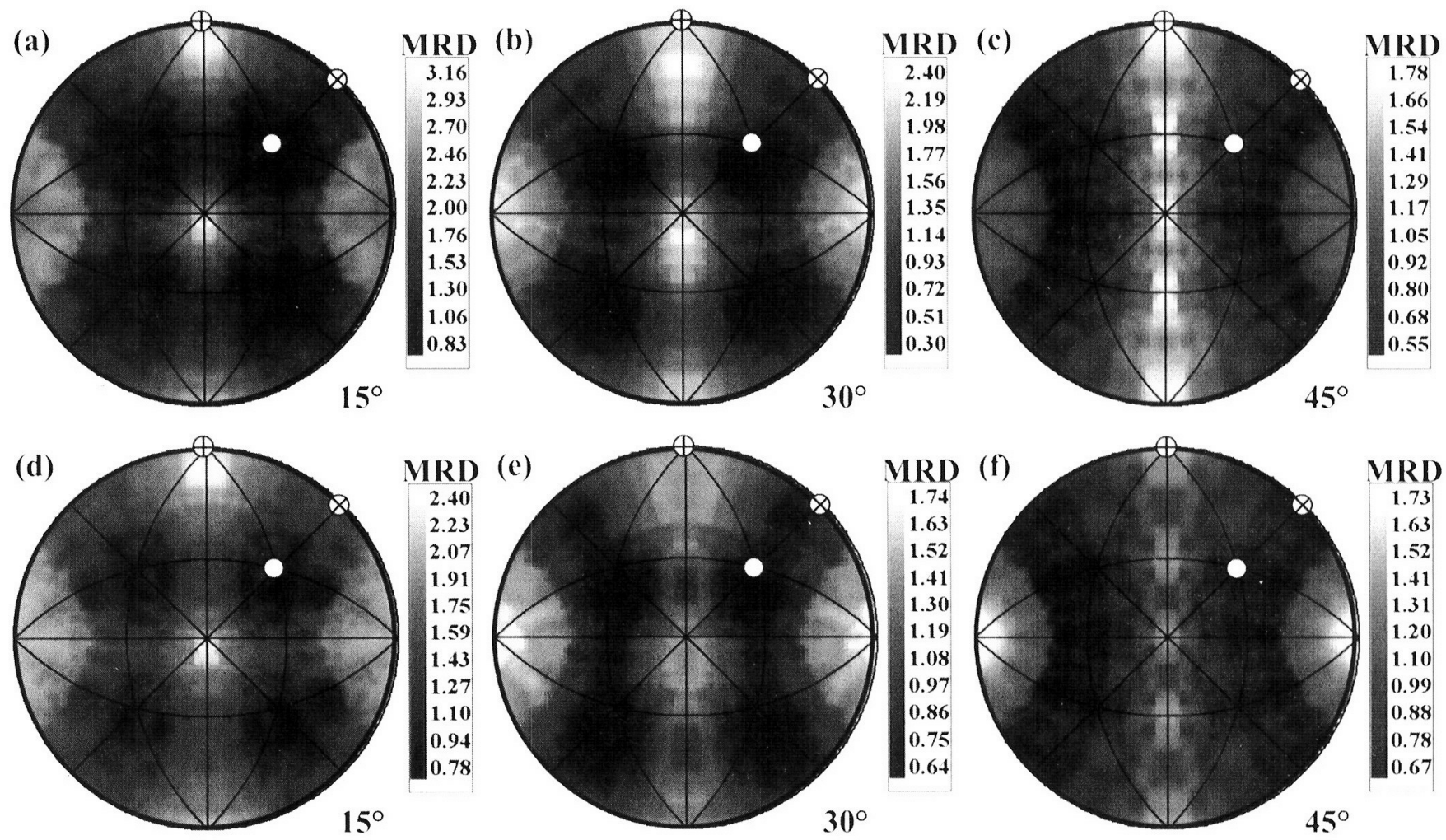

Fig. 5-The distribution of grain-boundary plane normals in $\mathrm{SrTiO}_{3}$, recovered using our method, for boundaries with misorientations of (a) 15 , (b) 30, and $(c) 45 \mathrm{deg}$ about [100]. $(d)$ through $(f)$ The distributions of plane normals observed in the serial sectioning experiment for the respective misorientations, shown for comparison. The populations, represented as MRD, are plotted in stereographic projection, with the [010], [110], and [111] directions marked with a circled "+," a circled " $\times$," and an open circle, respectively.

plane distribution for a 15 deg rotation about [100] that was estimated based on the section plane data only. We observe a strong preference for $\langle 100\rangle$ type boundary planes at that particular misorientation. For comparison, the boundary plane distribution at the same misorientation determined by direct measurements of the grain-boundary network is given in Figure $5(\mathrm{~d})$, which shows a similar preference for $\langle 100\rangle$ type planes. Additional plane distributions at misorientations corresponding to rotations of 30 and 45 deg about [100] are also shown in Figure 5. Although there are varying levels of consistency, these also show good agreement between the distribution estimated using our method and distribution determined with direct microstructural measurements. We again have the problem that it is unrealistic to visually examine the entire boundary space. Thus, we turn to quantifying the distribution of errors between the two boundary distributions, and we find that 95 pct of the errors are less than $0.81 \mathrm{MRD}$. This level of error is consistent with analyses of simulated data sets containing roughly the same number of obscrvations.

\section{DISCUSSION}

The results presented in Section IV demonstrate that $\lambda(\Delta g, \mathbf{n})$ can be determined from observations of a single planar section. The accuracy the grain-boundary distributions determined in this way depends on the number of observations considered. The results presented here show that reasonably accurate estimates of $\lambda(\Delta g, \mathbf{n})$ for materials with cubic symmetry can be obtatined using $5 \times 10^{4}$ or more observed boundary traces. While this represents a large quantity of microscopic data, it is now possible to collect such information in an automated fashion using orientation imaging microscopy. ${ }^{|6|}$

The only comparable experimental measurements of $\lambda(\Delta g, \mathbf{n})$ were made by analyzing parallel serial section planes produced by polishing. ${ }^{|8|}$ The method presented here provides some unique advantages over serial sectioning. First, data from only a single planar section are needed and this reduces the time spent collecting data. Furthermore, there are unavoidable errors when the geometry of the interfacial network is interpolated from data on parallel planes. Interfacial surfaces are approximated as planes and when a grain terminates within the removed section, connectivity is lost. Also, while the section planes are on average parallel, local fluctuations lead to geometric errors. Finally, the resolution of the sectioning process is usually far less than the microscopy, so that the errors associated with geometric measurements perpendicular to the section planes are at least an order of magnitude larger than errors parallel to the section plane. ${ }^{|8|}$ The geometric measurements used for the stereological analysis do not have the uncertainty added by the out-of-plane measurements.

Although the method described here can be used to extract boundary distributions with relative ease compared to other available experimental techniques, there are inherent errors. For example, errors arise because of the discrete nature of the approach. This source of crror was described in detail in a previous article. ${ }^{|3|}$ We also used an approximation to 
determine the total length of line incorrectly added to each cell in the accumulator. The values of $\omega_{i j}$ in Eq. $|1|$ decrease continuously in cells further and further from the boundary of interest. This continuous behavior was approximated as a step function with one term for nearest neighbor cells and another for all cells further removed. If we consider a maximum in the distribution, the incorrect line length from the near neighbor cells will be overestimated, while the incorrect line length from non-near neighbor cells is an underestimate. Also, for a minimum in the distribution, just the opposite occurs, where the incorrect length from the near neighbors is an underestimate and the contribution from the non-near neighbors is an overestimate. Thus, there is a partial cancellation of errors. Further, the magnitude of the errors is expected to increase with the magnitude of the anisotropy in the distribution. In other words, as the magnitude of the extrema in the distribution increases, the magnitude of the crrors associated with approximating the continous behavior of $\omega_{i j}$ as a step function, along with the corresponding errors in the incorrect line length estimation, will also increase. For example, although we have not tested a random distribution, we expect that the error between the recovered and actual distributions in such a case would be quite small. In fact, for a completely random distribution, the values in the accumulator will be identical in cach cell. Thus, the correction factor, and therefore, the final calculated frequency would also be the same in every cell. For a given level of discretization, it is possible to determine all of the weight factors, $\omega_{i j}$; it is then possible to solve the system of linear equations described by Eq. II for the correct line lengths in each cell, $l_{i}$. While the approximation described here appears satisfactory, we are currently attempting to determine the weighting factors more exactly to see if this leads to a significant reduction in error.

Errors in the recovered gralin-boundary distribution can also occur due to texture of the grain orientations. The technicjue described here is based on the assumption of random sampling of each grain-boundary type. In other words, we assume that regardless of the frequency of a specific boundary type, boundaries of that type are randomly orientated within the polycrystal. This is not the case for all polycrystalline materials. In materials with preferred grain oricntations, we observe many copies of the same sections, instead of randon sections, from each type of bicrystal. This biases the accumulation of line segment observations toward the texture axis. Research currently underway is aimed at quantifying and correcting for this elfect. Initial tests have shown that the performance of the method is not significantly affected if at least 60 pet of the grains in the microstructure are randomly distributed. In the case of a heavily textured polycrystal, the method will not work with observations from a single section plane. However, observations obtained from multiple randomly orientated section planes through the textured polycrystal would provide random sections from each grain-boundary type. While the process of making oblique sections adds complexity to the procedure, it is still less challenging than calibrated serial sectioning.

There are several differences between the stereological procedure described here and that previously used to determine the habits of embedded crystals. First, it should be noted that far fewer observations are needed to determine crystal habits, since the domain of possible bounding planes is two- dimensional rather than five-dimensional. Habits can be accurately determined based on that the approximately $10^{2}$ observations. The second difference is that the crystals were assumed to be fully faceted. As long as there is a limited number of well-defined facets, the origin of cach observed line segment can be assigned with reasonable confidence and area ratios can therefore be measured. In the present article, we have applied an approximate procedure to eliminate incorrectly assigned planes from the data set. By applying the new procedure to embedded crystals, even habits that include smoothly curving planes can be measured.

It is known that many grain-boundary sensitive properties, such as stress corrosion cracking, 1111 electrical activity, ${ }^{|12|}$ and creep behavior, ${ }^{|13|}$ are strongly influenced by the types of grain boundaries in a polycrystalline material and the way that they are connected. One application for the stereological method described here is to measure $\lambda(\Delta g, \mathbf{n})$ so that the performance of polycrystalline materials can be correlated to their grain-boundary distribution. Mcasurements of $\lambda(\Delta g, \mathbf{n})$ can also be used to study microstructural evolution. By quantifying the evolution of the population, it should be possible to gain some insight into the relative velocities of the diflerent boundary types. Finally, the method presented could be used to determine the crystallographic distribution of any type of randomly distributed interface.

\section{SUMMARY}

We have developed a method to estimate five-parameter grain-boundary distributions in polycrystals from a single observation plane. The method has been tested in two ways. First, simulated data sets based on known boundary distributions were analyzed. We found that, assuming cubic symmetry, the true grain-boundary distribution could be accurately estimated based on $5 \times 10^{4}$ or more observations. Second, grain-boundary distributions determined from a stereological analysis of a single plane of observations from a $\mathrm{SrTiO}_{3}$ polycrystal compared favorably to the distributions derived from the analysis of serial sections in the same sample. Thus, we conclude that the method described here provides a reasonable estimation of the boundary distribution using observations from a single planar section. The method should be valuable for the study of microstructural evolution and the relationships between microstructure and grain-boundary sensitive properties.

\section{ACKNOWLEDGMENTS}

One of the authors (DMS) thanks his colleagues at NIST, Edwin R. Fuller, Jr. and Grady S. White, for their helpful advice and criticisms, as well as Mark D. Vaudin and James A. Warren for their critical review of this manuscript. This work was supported at CMU by the MRSEC program of the National Science Foundation under Award No. DMR-0079996.

\section{REFERENCES}

1. H.F. Poulsen, S.F. Nielsen, E.M. Lauridsen, S. Schmidt, R.M. Suter, U. Lienert, L. Margulies, T. Lorentzen, and D. Juul Jensen: J. Appl. Cryst., 2001, vol. 34, pp. 751-56.

2. J. Alkemper and P.W. Voorhees: Acta Mater, 2000 , vol. 49, pp. 897-902. 
3. D.M. Saylor and G.S. Rohrer: J. Am. Cor. Soc, 2002, vol. 85, pp. 2799-2804.

4. B.L. Adams: Metall. Trans. A, 1980, vol. 17 A. pp. 2190-207.

5. R.J. Larsen and B.L. Adams: Proc, Plasticity '02, A.S. Khan and O. Lopez-Pamies, eds, Neat Press, Fulton, MD, 20(22, pp. 27-29.

6. B.I. Adams, S.I. Wright, and K. Kunze: Metall. Mater. Tiams. A, 1993 , vol. 24A, pp. 819-31

7. A. Morawiec: Proc: 3rd Int. Conf. on Grain Gronth. H. Weiland, B.I. Adams, and A.D. Rolletl, eds. TMS, Warrendale, PA, 1998 , pp. $509-14$.
8. D.M. Saylor, A. Morawiec, and G.S. Rohrer: Aeta Mater, 2003, vol. 51, pp. $3663-74$.

9. M.G. Kendall and P.A.P. Moran: Geometrical Probabilits. I latixer Publishing Company. New York, NY, 1963, pp. 78-80.

10. The naming of commercial eguipment does not imply endorsement by NIST.

11. G. Palumbo, E.M. Letockey, and P. I in: JoM, 1908, vol. 50, pp, 40-43.

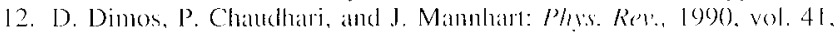
p). $4038-49$.

13. J. Don and S. Majumdar: Acta Mot., 1986, vol. 34, pp. 961-67. 